\title{
Afrontamiento y calidad de vida. Un estudio de pacientes con cáncer
}

\author{
Dario Martín Llull \\ Justo Zanier \\ Fernando García
}

\begin{abstract}
Resumen
Este trabajo es parte de un estudio longitudinal llevado a cabo en pacientes con cáncer y su objetivo es analizar la calidad de vida y su relación con las estrategias de afrontamiento. Se evidencia la necesidad de explorar y describir estos aspectos para obtener una mirada integradora del vivenciar de los enfermos neoplásicos, en función de un futuro trabajo interdisciplinario. Los sujetos fueron obtenidos en un hospital de Mar del Plata, Argentina. Fueron administrados un Cuestionario de Calidad de Vida, un Inventario de Respuestas de Afrontamiento y una entrevista semiestructurada que indaga variables sociodemográficas y psicológicas. Se muestran las relaciones entre las estrategias utilizadas por los pacientes para afrontar la enfermedad y su percepción de la calidad de vida. Son halladas fuertes correlaciones entre las estrategias de evitación y el deterioro en la mayoría de las dimensiones de la calidad de vida. Para concluir, se efectúan reflexiones e hipótesis al respecto.

Palabras-Clave: Calidad de vida; Afrontamiento; Psicooncología; Cáncer.
\end{abstract}

\section{Coping and quality of life. A study with patient with cancer}

\begin{abstract}
This study is part of a longitudinal research with oncologic patients and aims to analyze their quality of life and its relationship with coping. To explore and describe this aspects is important to give an integrative and interdisciplinary view about oncologic illness. The sample was gathered in an hospital of Mar del Plata, Argentina. A Quality of Life Questionnaire, a Coping Responses Inventory and a semi-structured interview were administered to collect information about the proposed variables, demographic and psychological characteristics. The results show the significance of relationship between coping with illness and the perceived quality of life, mainly between avoidance cognitive strategies and deterioration in the most of the dimension of the quality of life. Results and hypothesis proposed are discussed.
\end{abstract}

Keywords: Quality of life; Coping; Psycho-oncology; Cancer.

\section{Introducción}

En el campo de las enfermedades físicas y específicamente en la psicooncología la dificultad radica en establecer, dentro del amplio mundo de las variables psicológicas, cuáles tienen un mayor peso específico a la hora de predecir el tipo de patología física que puede tener un individuo concreto, o bien cuáles pueden predecir un mejor ajuste, calidad de vida y un mayor tiempo de supervivencia una vez establecida la enfermedad. Algunas de las variables psicológicas estudiadas son, por ejemplo, personalidad, afrontamiento, soporte social, stress, ansiedad, depresión, rotulación y evaluación del problema, por ejemplo (GrossarthMaticek, 2000; Schnoll, Harlow, Stolbach \& Brandt, 1998; Stiebler Leal, 1993).

Si a esto añadimos la necesidad de establecer vías de conexión entre los aspectos psico-sociales y los biológicos, comprenderemos que la dificultad es máxima y que, dada nuestra capacidad limitada de procesamiento, debemos contentarnos, hoy por hoy, con establecer relaciones empíricas entre algunas variables tanto biológicas, como psicológicas y sociales. Y es así que uno de los aspectos a estudiar que ha cobrado gran importancia el último tiempo es el afrontamiento.

Las estrategias de afrontamiento se refieren a cómo la gente se maneja ante las demandas de los sucesos estresantes. Son aquellos esfuerzos cognitivos y conductuales constantemente cambiantes que se desarrollan para manejar las demandas específicas externas $y / o$ internas que son evaluadas como excedentes o desbordantes de los recursos del individuo. Este modelo se denomina modelo transaccional del stress en tanto que el afrontamiento no es simplemente una respuesta a un hecho que ha sucedido, sino que es un proceso activo de modelado de lo que ha sucedido y de lo que sucederá.

Existen dos clasificaciones predominantes del afrontamiento. En una primera, se divide entre las formas centradas en el problema - dirigidas a manipular o alterar el problema, las centradas en la emoción dirigidas a regular la respuesta emocional que genera el

Endereço para correspondência:

${ }^{1}$ E-mail: dallull@mdp.edu.ar 
problema - y las centradas en la evaluación. Una segunda clasificación, según el foco, es la efectuada entre formas de afrontamiento activas (o de aproximación) versus formas de evitación. Las primeras, se refieren a aquellos esfuerzos cognitivos o conductuales para manejarse directamente con el evento estresante. Las formas evitativas consisten en el no enfrentamiento con el problema o en la reducción de la tensión a través de conductas de escape (Moos, 1998).

Se han publicado diversos estudios empíricos sobre estrategias de afrontamiento. Valderrama, Carbellido y Domínguez (1995) encontraron en 71 mujeres con cáncer cervicouterino que el estilo de afrontamiento más utilizado es la negación, aunque las pacientes hacen uso de una amplia gama de esfuerzos cognitivos y conductuales para controlar el problema y sus consecuencias emocionales. A su vez, Stiebler Leal (1993) constató una asociación significativa entre las respuestas psicológicas iniciales de las pacientes al diagnóstico y los resultados de la progresión de la enfermedad después de cinco, diez y quince años. Las consecuencias favorables fueron más frecuentes en pacientes cuyas respuestas fueron categorizadas como negación o espíritu de lucha, no así la aceptación estoica ni la desesperanza. En otro estudio con 65 pacientes con cáncer de mama no metastásico (Ferrero Berlanga, Toledo Aliaga \& Barreto Martín, 1995) el espíritu de lucha también se asoció significativamente con una mejor percepción global de la calidad de vida y menor distrés psicológico. El fatalismo, la preocupación ansiosa y el desamparo/ deseperanza se asociaran a una menor calidad de vida global y mayor distrés. Asimismo, en un estudio con 269 mujeres con cáncer genital y mamario encontraron una asociación entre mayores problemas psicosociales, mayor depresión y más utilización de estrategias de resignación y catarsis (Ibáñez \& Rosbundo, 1993).

$\mathrm{Al}$ mismo tiempo que atender al modo de afrontamiento, en el caso específico de los pacientes con cáncer, históricamente los objetivos de los tratamientos han sido reducir los síntomas y prolongar la vida (Stiebler Leal, 1993). Sin embargo, el tiempo de supervivencia no dice nada en cuanto a la calidad de ésta. Asimismo, los niveles de bienestar no dependen exclusivamente (ni siquiera fundamentalmente en algunos casos) de las variables físicas, sino también de las psicológicas y psicosociales, consideradas como codeterminantes de la calidad de vida.

Como creemos que el ser humano es un ser biopsicosocial e inserto en el tiempo y en la historia, lo que él piensa, lo que siente, lo que se imagina, tienen una importancia mucho mayor de lo que se presupone en el pronóstico y evolución de cualquier enfermedad. Estos aspectos se inscriben fundamentalmente en el concepto de "calidad de vida" (CV), entendido como

\begin{abstract}
la percepción de un individuo del lugar que ocupa en el entorno cultural y en el sistema de valores en que vive, asi como en relación con sus objetivos, expectativas, criterios y preocupaciones. Todo ello matizado, por supuesto, por su salud física, su estado psicológico, su grado de independencia, sus relaciones sociales, los factores ambientales y sus creencias personales. (OMS, 1996)
\end{abstract}

Teniendo en cuenta la concepción presentada de la CV, Bonicatto, Dew, Zaratiegui, Lorenzo y Pecina (2001), pioneros en la investigación sobre CV en Argentina administraron sobre diversas poblaciones el WHOQOL-100 (un instrumento desarrollado por la OMS para evaluar CV) y encontraron que la CV estaba significativamente deteriorada en pacientes depresivos, a diferencia de poblaciones con otras patologías crónicas severas como cáncer, dolor lumbar e hipertensión. Asimismo, analizando sólo los pacientes oncológicos, se evidenciaron menores puntajes en la dimensión psicológica, las relaciones sociales y la percepción del medio ambiente, a diferencia del aspecto físico y el nivel de independencia. Asimismo, Mor (1987) ha encontrado fuertes relaciones entre el estado físico y las mediciones en la $\mathrm{CV}$, proponiendo que una caída en los niveles del performance status está asociada a una baja significativa en la CV.

Debido a interrogantes que quedan pendientes a partir de los estudios publicados, se propone en el actual trabajo analizar la calidad de vida y su relación con las estrategias de afrontamiento, teniendo como hipótesis inicial que las estrategias de afrontamiento del paciente son una variable moduladora de la evaluación subjetiva de la CV. Se evidencia la necesidad de explorar y describir estos aspectos para obtener una mirada integradora del vivenciar de los enfermos neoplásicos, en función de un futuro trabajo interdisciplinario. Al mismo tiempo, tales evaluaciones pueden contribuir en una mayor adecuación de los abordajes terapéuticos empleados por los profesionales de la salud en la planificación de los servicios sanitarios y en la implementación de estrategias de prevención.

\section{Método}

\section{Participantes}

Se evaluaron 79 pacientes con cáncer del Hospital Interzonal General de Agudos de Mar del Plata que acudieron a su consulta con el oncólogo y a quienes se les indicó la realización de alguno de los siguientes tratamientos de quimioterapia, tales como, mama adyuvante $(n=29)$, mama avanzado $(n=14)$, pulmón avanzado $(\mathrm{n}=8)$, colon/recto $(\mathrm{n}=13)$ y otros $(n=15)$. Se realizó un estudio longitudinal, efectuando la misma evaluación de los pacientes en dos momentos, 
antes y después de la quimioterapia. La muestra presentó una media de 54.08 años, con una desviación standard de 8.3 y un rango de 34 a 77 años, siendo el $80.5 \%$ mujeres y el $19.5 \%$ hombres.

\section{Instrumentos}

Se administró la adaptación castellana del "Functional Assessment of Cancer Therapy Scale" (FACT) para evaluar la Calidad de Vida (Cella \& col., 1993). Es un cuestionario autoadministrado que evalúa cinco dimensiones desde el punto de vista subjetivo del paciente, cuales sean, estado físico general de salud, ambiente familiar y social, relación con el médico, estado emocional, capacidad de funcionamiento personal, y otras preocupaciones asociadas a la enfermedad.

Fue utilizada la adaptación castellana del "Inventario de Respuestas de Afrontamiento" (CRI) (Moos, 1990). Este cuestionario autoadministrado categoriza las respuestas de afrontamiento en ocho tipos específicos, según se indica en la Tabla 1.

Tabla 1 - Clasificación de las estrategias de afrontamiento evaluadas por el CRI

Aproximación Evitación

Análisis Lógico (AL)

Evitación Cognitiva (EC)

Cognitivo

Revalorización Positiva (RP)

Aceptación o Resignación (AA)

\begin{tabular}{lll}
\hline Búsqueda de Apoyo y Orientación (BS) & $\begin{array}{l}\text { Búsqueda de Gratificaciones } \\
\text { Alternativas (BR) }\end{array}$
\end{tabular}

Conductual

Resolución de Problemas (RPR) Descarga Emocional (DE)

Como se puede observar, las ocho estrategias de afrontamiento que evalúa el CRI se pueden clasificar según el método (cognitivo vs. conductual) y según el foco (aproximación vs. evitación). A su vez, se pueden diferenciar en las que son centradas en el problema (búsqueda de sostén, resolución de problemas y búsqueda de recompensas alternativas), centradas en la emoción (descarga emocional y aceptación pasiva/ resignación) y centradas en la evaluación (análisis lógico, revalorización positiva y evitación cognitiva).

\section{Procedimientos}

Se sistematizaron y codificaron los datos y se realizaron análisis estadísticos descriptivos de cada una de las variables involucradas en el estudio. Se estimaron coeficientes de correlación entre las variables, diferencias de puntajes medios (prueba t-test) y se efectuaron cálculos de regresión lineal, utilizando el software del paquete estadístico para ciencias sociales SPSS.

\section{Resultados}

Para el análisis de las estrategias de afrontamiento se consideraron los puntajes de cada una de las ocho escalas del CRI y los puntajes de estas escalas clasificadas según el foco, el método y hacia dónde está centrado el afrontamiento. La muestra total presentó, al iniciar la quimioterapia, las estrategias de afrontamiento que se muestran en la Tabla 2. 
Tabla 2 - Medias y desvío estándar de las estrategias de afrontamiento al inicio de la quimioterapia

\begin{tabular}{|c|c|c|c|c|c|c|c|c|c|c|c|c|}
\hline \multirow[t]{2}{*}{ Afrontamiento } & \multicolumn{2}{|c|}{$\begin{array}{l}\text { Muestra total } \\
\quad(n=79)\end{array}$} & \multicolumn{2}{|c|}{$\begin{array}{c}\text { Mama } \\
\text { adyuvante } \\
(\mathrm{n}=29)\end{array}$} & \multicolumn{2}{|c|}{$\begin{array}{c}\text { Mama } \\
\text { avanzado } \\
(\mathrm{n}=14)\end{array}$} & \multicolumn{2}{|c|}{$\begin{array}{l}\text { Pulmón } \\
\text { avanzado } \\
(\mathrm{n}=8)\end{array}$} & \multicolumn{2}{|c|}{$\begin{array}{l}\text { Cólon/Recto } \\
\qquad(n=13)\end{array}$} & \multicolumn{2}{|c|}{$\begin{array}{l}\text { Otros } \\
(n=15)\end{array}$} \\
\hline & Media & DS & Media & DS & Media & DS & Media & DS & Media & DS & Media & DS \\
\hline$\overline{\mathrm{AL}}$ & 4.94 & 4.35 & 5.45 & 3.97 & 4.93 & 5.33 & 4.88 & 5.19 & 5.08 & 5.19 & 3.87 & 3.07 \\
\hline $\mathrm{RP}$ & 10.68 & 4.49 & 9.72 & 4.60 & 11.64 & 4.50 & 10.38 & 5.01 & 11.31 & 4.68 & 11.27 & 4.06 \\
\hline BS & 9.16 & 3.68 & 9.17 & 3.88 & 9.71 & 2.76 & 6.38 & 3.70 & 10.46 & 4.52 & 9.00 & 2.73 \\
\hline RPR & 8.46 & 4.38 & 7.83 & 4.35 & 9.43 & 3.30 & 7.50 & 4.63 & 8.38 & 5.32 & 9.33 & 4.56 \\
\hline $\mathrm{EC}$ & 7.89 & 3.80 & 7.83 & 3.28 & 8.50 & 4.82 & 7.00 & 4.11 & 7.85 & 3.00 & 7.93 & 4.53 \\
\hline $\mathrm{AA}$ & & 4.03 & 6.07 & 4.17 & 5.64 & 4.52 & 7.63 & 4.63 & 7.62 & 3.25 & 5.20 & 3.53 \\
\hline BR & 6.75 & 4.22 & 6.00 & 4.12 & 8.14 & 3.74 & 7.75 & 5.87 & 6.62 & 4.54 & 6.47 & 3.66 \\
\hline $\mathrm{DE}$ & 4.35 & 3.47 & 4.72 & 3.24 & 4.21 & 4.41 & 5.25 & 3.58 & 2.77 & 3.27 & 4.67 & 3.04 \\
\hline Aproximación & 8.31 & 3.34 & 8.04 & 3.34 & 8.92 & 2.74 & 7.28 & 4.28 & 8.80 & 4.14 & 8.36 & 2.78 \\
\hline Evitación & 6.30 & 2.36 & 6.15 & 2.29 & 6.62 & 3.05 & 6.90 & 2.92 & 6.21 & 2.23 & 6.06 & 1.75 \\
\hline Cognitivo & 7.43 & 2.49 & 7.26 & 2.56 & 7.67 & 2.52 & 7.46 & 2.61 & 7.96 & 3.00 & 7.06 & 1.99 \\
\hline Conductual & 7.18 & 2.92 & 6.93 & 2.90 & 7.87 & 2.56 & 6.71 & 3.91 & 7.05 & 3.53 & 7.36 & 2.34 \\
\hline Aprox Cognitiva & 7.81 & 3.66 & 7.58 & 3.54 & 8.28 & 3.44 & 7.62 & 4.88 & 8.19 & 4.43 & 7.56 & 3.03 \\
\hline Aprox Conductual & 8.81 & 3.57 & 8.50 & 3.69 & 9.57 & 2.40 & 6.93 & 3.88 & 9.42 & 4.55 & 9.16 & 3.17 \\
\hline Evitac Cognitiva & 7.06 & 3.20 & 6.94 & 3.21 & 7.07 & 3.83 & 7.31 & 3.24 & 7.73 & 2.32 & 6.56 & 3.50 \\
\hline Evitac Conductual & 5.55 & 3.06 & 5.36 & 2.78 & 6.17 & 3.52 & 6.50 & 4.61 & 4.69 & 3.11 & 5.56 & 2.18 \\
\hline Problema & 8.12 & 3.39 & 7.66 & 3.40 & 9.09 & 2.46 & 7.20 & 4.16 & 8.48 & 4.21 & 8.26 & 3.05 \\
\hline Emoción & 5.29 & 2.82 & 5.39 & 3.08 & 4.92 & 3.18 & 6.43 & 3.23 & 5.19 & 2.52 & 4.93 & 2.03 \\
\hline Evaluación & 7.83 & 2.81 & 7.66 & 2.62 & 8.35 & 3.09 & 7.41 & 3.02 & 8.07 & 3.50 & 7.68 & 2.42 \\
\hline
\end{tabular}

Los pacientes presentan más estrategias de aproximación que de evitación y más centradas en el problema y en la evaluación que en la emoción. Utilizan en su mayoría las estrategias aproximativas, salvo el "análisis lógico" (aproximación) que junto a la "descarga emocional" (evitación) son las dos menos utilizadas. Siendo el cáncer una enfermedad con un compromiso físico muy importante y una connotación asociada a una situación "terminal", es destacable que el recurso de afrontamiento más utilizado por los pacientes es la "revalorización positiva".

Se indagaron las diferencias en los puntajes medios (t-test) del CRI en relación a una serie de variables obteniendo que:

1. Las mujeres presentaron más recursos de "descarga emocional" que los hombres (Dif. Media $=2.43$, $\mathrm{p}<.01$, Std. Error Difference=0.80). Probablemente esto se deba a la mayor libertad que en nuestro medio cultural se otorguen las mujeres en expresar sus emociones, llorar, etc.

2. Se particionó al grupo en dos según la edad (con línea de corte en 55 años) y se encontró que las personas con 55 o más años utilizan en mayor medida que los más jóvenes las estrategias de búsqueda de sostén (dif. media $=1.89, \quad \mathrm{p}<.05, \quad$ std. error difference $=0.81$ ), resolución de problemas (rpr: dif. media $=2.28, \quad \mathrm{p}<.05$, std. error difference $=0.95)$, descarga emocional (dif. media $=2.85, \mathrm{p}<.001$, std. error difference $=0.72$ ), aproximación (dif. media $=1.82, \mathrm{p}<.05$, std. error difference $=0.73$ ), afrontamiento conductual (dif. media $=2.13 \mathrm{p}<.001$, std. error difference $=0.61$ ) y centrado en el problema (dif. media $=1.89 \mathrm{p}<.05$, std. error difference $=0.73$ ).

3. Se encontraron diferencias significativas en el afrontamiento de personas con diversos niveles educativos. Se particionó al grupo en dos, hasta primario incompleto vs. desde secundario incompleto en adelante, y los integrantes de este último grupo presentaron mayores puntajes en casi la totalidad de las estrategias, tales como análisis lógico (dif. media $=4.60$, $\mathrm{p}<.001$, std. error difference $=0.93$ ), revalorización positiva (dif. media $=4.02, \mathrm{p}<.001$, std. error difference $=0.93$ ), búsqueda de sostén y orientación (dif. media $=3.05 \mathrm{p}<.01$, std. error difference $=0.95$ ), resolución de problemas (dif. media $=4.56 \mathrm{p}<.001$, std. error difference 0.96), búsqueda de recompensas alternativas (dif. media $=3.64, \mathrm{p}<.001$, std. error difference $=0.99)$ y descarga emocional (dif. media $=2.02$, $\mathrm{p}<.05$, std. error difference $=0.84$ ). Parecería que los pacientes con más nivel educativo dispondrían de mayor riqueza y variedad de recursos para afrontar las situaciones importantes o estresantes que se le presentaran.

4. Se encontró que los pacientes que manifestaron que consultarían a un psicólogo por algún motivo, tienen más puntajes en "análisis lógico" que los restantes (dif. media $=2.10, \mathrm{p}<.05$, std. error 
difference=0.96). El que utilicen recursos cognitivos con el fin de comprender y prepararse mentalmente para afrontar los estresores, podría ser una de las explicaciones por la cuales se muestren dispuestos a un espacio psicoterapéutico como modo de afrontar las situaciones que se les presentan y sus consecuencias.
En el plan de trabajo de esta investigación se planteó como hipótesis inicial que las estrategias de afrontamiento del paciente son una variable moduladora de la evaluación subjetiva de la calidad de vida. Y es precisamente el análisis correlacional del afrontamiento y la CV el que arroja los conocimientos más interesantes de este estudio.

Tabla 3-Coeficientes de correlación ( $\mathrm{r}$ de Pearson) entre Afrontamiento y Calidad de Vida, pre y post quimioterapia

\begin{tabular}{|c|c|c|c|c|c|c|c|c|}
\hline \multirow[b]{2}{*}{ AFRONTAMIENTO } & \multirow[b]{2}{*}{ MOMENTO } & \multicolumn{6}{|c|}{ CALIDAD DE VIDA } & \multirow[b]{2}{*}{ TOTAL } \\
\hline & & 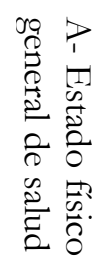 & 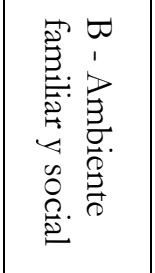 & 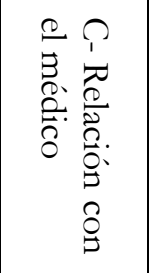 & 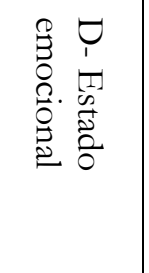 & 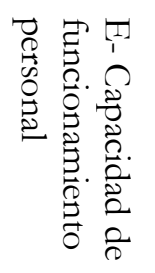 & 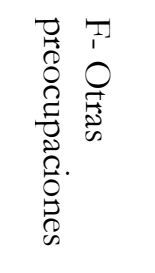 & \\
\hline Análisis Lógico & $\begin{array}{l}\text { Pre-Qt } \\
\text { Post-Qt }\end{array}$ & & & & $-0.326^{*}$ & & $-0.409 *$ & \\
\hline Revalorización Positiva & $\begin{array}{l}\text { Pre-Qt } \\
\text { Post-Qt }\end{array}$ & & & & & & $0.526^{* *}$ & \\
\hline $\begin{array}{l}\text { Búsqueda de Sostén y } \\
\text { Orientación }\end{array}$ & $\begin{array}{l}\text { Pre-Qt } \\
\text { Post-Qt }\end{array}$ & & & & & $0.333^{*}$ & & \\
\hline Resolución de Problemas & $\begin{array}{l}\text { Pre-Qt } \\
\text { Post-Qt }\end{array}$ & & & & & & $0.433^{*}$ & \\
\hline Evitación Cognitiva & $\begin{array}{l}\text { Pre-Qt } \\
\text { Post-Qt }\end{array}$ & & $\begin{array}{l}-0.342^{*} \\
-0.420^{*} \\
\end{array}$ & & $\begin{array}{c}-0.288 * \\
-0.509 * *\end{array}$ & & $\begin{array}{c}-0.422^{*} \\
-0.529 * *\end{array}$ & $-0.428^{* *}$ \\
\hline $\begin{array}{l}\text { Aceptación pasiva, } \\
\text { resignación }\end{array}$ & $\begin{array}{l}\text { Pre-Qt } \\
\text { Post-Qt }\end{array}$ & & $\begin{array}{l}-0.378^{*} \\
-0.350^{*}\end{array}$ & $\begin{array}{l}-0.349 * \\
-0.539 * *\end{array}$ & $\begin{array}{l}-0.331 * \\
-0.564 * *\end{array}$ & $\begin{array}{l}-0.439 * * \\
-0.466 * *\end{array}$ & $\begin{array}{l}-0.442^{*} \\
-0.392 *\end{array}$ & $\begin{array}{l}-0.438 * * \\
-0.488 * *\end{array}$ \\
\hline $\begin{array}{l}\text { Búsqueda de } \\
\text { recompensas alternativas }\end{array}$ & $\begin{array}{l}\text { Pre-Qt } \\
\text { Post-Qt }\end{array}$ & & $0.415^{*}$ & $0.365^{*}$ & & & & \\
\hline Descarga Emocional & $\begin{array}{l}\text { Pre-Qt } \\
\text { Post-Qt }\end{array}$ & & $-0.397 * *$ & & $\begin{array}{c}-0.335^{*} \\
-0.628^{* *}\end{array}$ & $-0.416^{*}$ & $-0.490 * *$ & $\begin{array}{l}-0.445^{* *} \\
-0.511 * *\end{array}$ \\
\hline APROXIMACIÓN & $\begin{array}{l}\text { Pre-Qt } \\
\text { Post-Qt }\end{array}$ & & & & & & $0.366^{*}$ & \\
\hline EVITACIÓN & $\begin{array}{l}\text { Pre-Qt } \\
\text { Post-Qt }\end{array}$ & & $-0.425^{* *}$ & $-0.322 *$ & $\begin{array}{c}-0.350 * \\
-0.634 * *\end{array}$ & & $\begin{array}{l}-0.440^{*} \\
-0.565^{* *}\end{array}$ & $\begin{array}{l}-0.389 * * \\
-0.407 *\end{array}$ \\
\hline COGNITIVO & $\begin{array}{l}\text { Pre-Qt } \\
\text { Post-Qt }\end{array}$ & & & & $\begin{array}{l}-0.313^{*} \\
-0.555^{* *}\end{array}$ & & $-0.528^{* *}$ & $-0.373^{*}$ \\
\hline CONDUCTUAL & $\begin{array}{l}\text { Pre-Qt } \\
\text { Post-Qt }\end{array}$ & & & & & & & \\
\hline $\begin{array}{l}\text { APROXIMACIÓN } \\
\text { COGNITIVA }\end{array}$ & $\begin{array}{l}\text { Pre-Qt } \\
\text { Post-Qt }\end{array}$ & & & & & & & \\
\hline $\begin{array}{l}\text { APROXIMACIÓN } \\
\text { CONDUCTUAL }\end{array}$ & $\begin{array}{l}\text { Pre-Qt } \\
\text { Post-Qt }\end{array}$ & & & & & & $0.406^{*}$ & \\
\hline $\begin{array}{l}\text { EVITACIÓN } \\
\text { COGNITIVA }\end{array}$ & $\begin{array}{l}\text { Pre-Qt } \\
\text { Post-Qt }\end{array}$ & & $\begin{array}{l}-0.429 * * \\
-0.467 * *\end{array}$ & $-0.360 *$ & $\begin{array}{l}-0.369 * \\
-0.644 * *\end{array}$ & $\begin{array}{l}-0.351 * \\
-0.460^{* *}\end{array}$ & $\begin{array}{l}-0.504 * * \\
-0.550 * *\end{array}$ & $\begin{array}{l}-0.420^{* *} \\
-0.550^{* *}\end{array}$ \\
\hline $\begin{array}{l}\text { EVITACIÓN } \\
\text { CONDUCTUAL }\end{array}$ & $\begin{array}{l}\text { Pre-Qt } \\
\text { Post-Qt }\end{array}$ & & & & $-0.402^{*}$ & & $-0.396^{*}$ & \\
\hline
\end{tabular}

${ }^{*} \mathrm{p}<0.05 /{ }^{* *} \mathrm{p}<0.01 /$ Pre-Quimioterapia $(\mathrm{n}=44) /$ Post-Quimioterapia $(\mathrm{n}=35)$ 
Por ello, para los datos obtenidos previo a la quimioterapia se realizó el análisis correlacional de este apartado con los individuos que respondieron al CRI identificando como "problema importante o estresante" a la enfermedad $(\mathrm{n}=44$, correspondiente al $55.7 \%$ del total). (Es de destacar que en la consigna original del CRI cada paciente debe identificar el problema más importante durante los últimos doce meses y luego responder al cuestionario). Para los datos post quimioterapia se tomaron todos los casos, dado que la consigna del CRI se modificó indicando que respondieran específicamente "en función de la enfermedad por la cual estaban en el hospital en ese momento" $(n=35)$. Teniendo en cuenta estas observaciones, se presentan en la Tabla 3 los coeficientes de correlación con significatividad estadística ( $\mathrm{r}$ de Pearson) entre el afrontamiento y la CV para cada uno de los dos momentos (pre y post-quimioterapia).

Se puede observar que los coeficientes de correlación obtenidos previo al inicio de la quimioterapia son similares, salvo pequeñas variaciones, con los arrojados al finalizar el tratamiento. Esto podría hablar de la significatividad de dicha relación. Las estrategias de evitación correlacionan, en su mayoría, negativamente con la CV. La evitación cognitiva (evitación cognitiva propiamente dicha y aceptación pasiva/resignación) presenta altos coeficientes de correlación con el puntaje total de la CV y la mayoría de las escalas. De la evitación conductual, específicamente la descarga emocional, también correlaciona negativamente.

Las únicas escalas que se asocian positivamente con algunos pocos dominios del FACT son la revalorización positiva (aproximación cognitiva), la búsqueda de sostén y la resolución de problemas (ambas de aproximación conductual) y la búsqueda de recompensas alternativas (evitación conductual). Además, es notable que las estrategias de afrontamiento no se asocian, en ningún sentido, con la evaluación que hacen los pacientes de su "estado físico general de salud".

Posteriormente a este análisis se efectuaron cálculos de regresión lineal para estimar qué dimensiones del afrontamiento podrían ser buenos predictores de la CV en este tipo de pacientes. En los resultados que se muestran se consideraron los datos del afrontamiento y la CV medidos después de finalizada la quimioterapia, considerando que el cuestionario de afrontamiento evalúa restrospectivamente las estrategias utilizadas por el paciente con respecto a la enfermedad y el cuestionario de CV mide cómo se encontró el sujeto durante los últimos siete días al momento de la administración (finalización de la quimioterapia).

La Tabla 4 muestra que el afrontamiento evitativo cognitivo y conductual predicen desde el $12 \%$ al $39 \%$ de la varianza de las dimensiones de la CV. Considerando el foco del afrontamiento, el centrado en la emoción es el que presenta mayor valor predictivo, llegando a explicar hasta el $42 \%$ de la varianza del estado emocional.

Tabla 4 - Regresión lineal. El Afrontamiento como predictor de la Calidad de Vida

\begin{tabular}{llcc}
\hline Predictor & Dimensión de la calidad de vida & $\mathrm{R} 2$ & $\mathrm{~F}$ \\
\hline Evitación cognitiva & CV Total & $0.28^{* * *}$ & 14,292 \\
& Ambiente fliar. y social & $0.19^{* *}$ & 9,208 \\
& Estado emocional & $0.39^{* * *}$ & 23,438 \\
& Capac. de funcionam. personal & $0.18^{* *}$ & 8,871 \\
& Otras preocupaciones & $0.27^{* *}$ & 11,697 \\
\hline Evitación conductual & Estado emocional & $0.13^{*}$ & 6,375 \\
& Otras preocupaciones & $0.12^{*}$ & 5,035 \\
\hline Afrontamiento centrado en la emoción & Ambiente fliar. y social & $0.11^{*}$ & 5,283 \\
& Relación con el médico & $0.17^{* *}$ & 8,381 \\
& Estado emocional & $0.42^{* * *}$ & 25,894 \\
& Capac. de funcionam. personal & $0.22^{* *}$ & 10,666 \\
& Otras preocupaciones & $0.21^{* *}$ & 8,653 \\
& CV Total & $0.28^{* *}$ & 14,853 \\
\hline Afrontamiento centrado en el problema & Ambiente fliar. y social & $0.14^{*}$ & 6,659 \\
\hline Afrontamiento centrado en la evaluación & Estado emocional & $0.17^{* *}$ & 8,318 \\
& Capac. de funcionam. personal & $0.24^{* *}$ & 9,938 \\
\hline
\end{tabular}

${ }_{* * *} \mathrm{p}<0.001 /{ }^{* *} \mathrm{p}<0.01 / * \mathrm{p}<0.05$ 


\section{Discusión y Conclusiones}

Como objetivo general del estudio se esperó adquirir conocimientos que puedan enriquecer el enfoque psicológico del cáncer aportando, desde una perspectiva biopsicosocial, a los avances de otras disciplinas. Junto al gran desarrollo tecnológico y médico respecto a las enfermedades oncológicas, es imperiosa la necesidad de generar conocimientos interdisciplinarios para efectuar un abordaje integral del ser humano.

Como parte de este recorrido, hemos hallado que las estrategias de evitación hacia la enfermedad correlacionan negativamente con la percepción de la CV $\mathrm{y}$ predicen un alto porcentaje de su varianza. Estos resultados coincidirían con varios estudios realizados donde se encontró que las estrategias de aceptación estoica y desesperanza, que serían estrategias de evitación, implican consecuencias desfavorables en los pacientes (Stiebler Leal, 1993), el fatalismo y la desesperanza se asoció a una disminución de la CV global (Ferrero \& col., 1995), y las estrategias de resignación y catarsis (evitación cognitiva y conductual respectivamente) correlacionó con mayor deterioro psicosocial (Ibáñez \& Rosbundo, 1993). A su vez, a diferencia de la investigación de Valderrama y colaboradores (1995), donde se encontró la negación como afrontamiento más utilizado, en la población de pacientes estudiada en este trabajo las estrategias de aproximación fueron las más empleadas.

Además de la sintomatología física (enfermedad), es necesario considerar el padecimiento de los pacientes con cáncer - en la evaluación, pronóstico y tratamiento - desde una perspectiva humana integral. El médico realiza una labor increíblemente valiosa, pero junto a él, se necesitan otros profesionales especializados que efectúen intervenciones con los pacientes y sus familiares. Por ello, es prometedor el progreso en el diseño de programas de intervención interdisciplinaria (o al menos multidisciplinaria) que atiendan cada vez más las necesidades de pacientes y familiares con cáncer. De estos datos se deduce la necesidad de una intervención psicológica en los pacientes que desarrollen un afrontamiento que se asocia a mayor malestar y deterioro de la CV.

Es así como, a partir de las conclusiones de este trabajo, hemos diseñado un programa de psicoterapia grupal para pacientes con cáncer, en doce encuentros, desde un modelo cognitivo social. Inicialmente se está aplicando en grupos de pacientes con cáncer de mama que efectúan quimioterapia adyuvante simultáneamente. Carecemos de diseños empíricamente validados en nuestro país y por ello es esperable que estos estudios produzcan aportes en este sentido.

\section{Referencias}

Bonicatto, S., Dew, M. A., Zaratiegui, R., Lorenzo, L. \& Pecina, P. (2001). Pacientes de ambulatorios con depresión: peor calidad de vida que en otras enfermedades médicas en la Argentina. Social Science and Medicine, 52, 911-919.

Cella, D. \& col. (1993). The Functional assessment on Cancer Therapy Scale: Development and validation of the general measure. Journal of Clinical Oncology, 11, 570-579.

Ferrero Berlanga, J., Toledo Aliaga, M. \& Barreto Martín, M. (1995). Evaluación cognitiva y afrontamiento como predictores del bienestar futuro de las pacientes con cáncer de mama. Revista Latinoamericana de Psicología, 27(1), 87-102.

Grossarth-Maticek, R. (2000). Interaction of psychosocial and physical risk factors in the causation of mammary cancer, and its prevention through psychological methods of treatment. Journal of Clinical Psychology, 56(1), 33-41.

Ibáñez, E. \& Rosbundo, A. (1993). Soporte social y afrontamiento afectivo en la enfermedad cancerosa. Em Páez Rovira, D. Salud, expresión y represión social de las emociones. (p. 379-397). Valencia: Ed. Promolibro.

Moos, R. H. (1990). Coping Responses Inventory Manual. California, Center for Health Care Evaluation, Stanford University and Dept. Veterans' Administration Medical Centers.

Moos, R. H. (1998). Life Stressors and Coping Resources Influence Health and Well-Being. Psychological Assessment, 4(2), 133-158.

Mor, V. (1987). Cancer patients' Quality of Life over the disease course: lessons from the real world, Journal of Chronical Disease, 40(6), 535-544.

OMS, Grupo sobre calidad de vida (1996). ¿Qué calidad de vida? Foro Mundial de la Salud, 17, 385-387.

Schnoll, R., Harlow, L., Stolbach, L. \& Brandt, U. (1998). A structural model of the relationships among stage of disease, age, coping, and psychological adjustment in women with breast cancer. Psycho-Oncology, 7, 69-77.

Stiebler Leal, V. (1993). Variáveis psicológicas influenciando o risco e o prognóstico do cancer: um panorama atual. Revista Brasileira de Cancerologia, 39(2), 53-59.

Valderrama Iturbe, P., Carbellido Vásquez, S. \& Domínguez-Trejo, B. (1995). Ansiedad y Estilos de Afrontamiento en mujeres con cáncer cervicouterino. Rev. Latinoamericana de Psicología, 27(1), 73-86. 
Recebido em junbo de 2003

Reformulado em novembro de 2003 Aprovado em dezembro de 2003

Sobre os autores:

Darío M. Llull é psicólogo, professor e pesquisador na Facultad de Psicología, Universidad Nacional de Mar del Plata e no curso de Ciências da Educação, e membro da Fundación Aiglé.

Justo Zanier é doutor em Medicina, diretor do Instituto de Genética Humana, AGHU, Mar del Plata, professor titular e pesquisador da Universidad Nacional de Mar del Plata e coordenador do programa de bioética da Universidad Nacional de Mar del Plata.

Fernando García é doutor em Psicología e professor da Facultad de Psicología da Universidad Nacional de Mar del Plata, atua como professor de graduação e pós-graduação da Universidad de Belgrano, Argentina e como professor e coordenador de pesquisa da Fundación Aiglé. 